\title{
EI modelo de salarización en el mercado laboral gallego: Influencia del género
}

\author{
Ma Carmen Sánchez-Sellero ${ }^{1}$, Pedro Sánchez-Sellero ${ }^{2}$ \\ ${ }^{1}$ Universidad de La Coruña, ${ }^{2}$ Universidad de Zaragoza (Spain) \\ c.sanchez@udc.es,pedross@unizar.es
}

Received May, 2013

Accepted August, 2013

\section{Resumen}

Objeto: Se estudian las características personales que determinan la participación en el mercado de trabajo en Galicia, ya sea como trabajador por cuenta propia o por cuenta ajena.

Diseño/metodología: Se construye el llamado modelo de salarización, en él se estima la probabilidad de que una persona en edad de trabajar lo haga por cuenta ajena. Una vez estimados los parámetros del modelo mediante regresión logística binaria, se calculan las probabilidades de trabajar por cuenta ajena condicionadas a distintas características personales, para evaluar la influencia real de las mismas en la forma en que un individuo interviene en el mercado de trabajo, como asalariado o como autónomo.

Aportaciones $\boldsymbol{y}$ resultados: Las probabilidades de trabajar por cuenta ajena difieren en función del género. A mayor edad, la probabilidad de que un hombre y una mujer trabajen por cuenta ajena va disminuyendo. En los hombres, la probabilidad de trabajar por cuenta ajena se mantiene invariable para cualquier tamaño familiar, mientras que en las mujeres al aumentar los miembros de la familia la probabilidad disminuye. Ante un incremento en los ingresos corregidos familiares, la probabilidad de trabajar por cuenta ajena disminuye en hombres y mujeres, aunque los porcentajes son distintos. 
Limitaciones: Este trabajo ha sido realizado en un momento económico de crecimiento sostenido. Así, se abre una futura línea de estudio en la que se averigüe el efecto del ciclo económico en esta investigación.

Implicaciones prácticas: El género sigue marcando diferencias en el mercado laboral.

Valor añadido: En este análisis, la variable más destacada es el sexo, y la influencia de las demás variables explicativas sobre las distintas probabilidades de trabajar por cuenta ajena varía en función del sexo de la persona analizada. La forma de construir la variable "Ingresos del hogar corregidos" es novedosa.

Palabras clave: Mercado laboral, Logit, Empleador, Género

Códigos JEL: C13, C25, J01, J23, J71

Title: The wage model in the Galician labour market. Gender effects

\section{Abstract}

Purpose: We study the effects of personal features in the Galician labour market, as both self-employed people and employees who work for a firm.

Design/methodology/approach: We propose a wage model to estimate the probability of a person in working age being employee who works for a firm. After the model parameter estimation through binary logistic regression, we estimate the conditional probability to be an employee who works for a firm for different personal features. We do this to evaluate the real effect of personal features in the person role in the labour market, as employee who works for a firm or self-employed person.

Findings and originality/value: The probability to be an employee who works for a firm depends on the gender. The greater age, the lower is the probability of men and women that they will be employees who work for a firm. The probability of men to work for a firm is the same to all family size, whereas this probability for women is lower if the family size gests bigger. The greater corrected family income, the lower is the probability of men and women to work for a firm, but the percentages are different.

Research limitations/implications: This paper has been made during a moment of sustained economic growth. So, future researches could study the effect of the economic cycle in this topic.

Practical implications: The gender differences affects to the labour market. 
Originality/value: This study considers as the most important variable the gender, and the effect of other explanatory variables on the different probabilities to be an employee who works for a firm change depending on the gender. We propose a new way to create the variable "corrected household income".

Keywords: Labour market, Logit, Employer, Gender

Jel Codes: C13, C25, J01, J23, J71

\section{Introducción}

El mercado de trabajo hoy en día está en boca de expertos, analistas, estudiosos de los distintos medios políticos, sociales, económicos y de comunicación. En la actualidad vivimos una situación económica afectada por una fuerte recesión e inestabilidad, además de una continua fase de reforma del mercado de trabajo. El momento actual genera en la opinión pública mucha expectación, así como preocupación y sensibilidad por las dudas de si este país saldrá y cuándo de la crisis en la que se ve inmersa.

Nuestro estudio del mercado de trabajo no va a trasladarse en el tiempo, con lo cual aunque este análisis tanto estadístico como económico es estático, no hemos querido pasar por alto la realidad tan difícil que nos ocupa y que tantas opiniones, encontradas a veces, está suscitando.

En el análisis empírico utilizaremos datos de la Comunidad Autónoma Gallega, concretamente microdatos de la "Encuesta de condiciones de vida de las familias" facilitados por el Instituto Galego de Estatística (IGE), con lo cual todos nuestros resultados y conclusiones van a referirse a esta Comunidad aunque podrían extrapolarse a un ámbito mayor. Creemos que las conclusiones pueden ser igualmente válidas en el ámbito nacional y siempre que la situación económica sea estable.

Este estudio se extrae de los modelos del mercado de trabajo recogidos en Sánchez Sellero (2010). Nos interesa conocer cuáles son los factores por los cuales una persona que ya forma parte del mercado de trabajo lo haga o bien como trabajador por cuenta ajena o bien por cuenta propia.

Una de las principales ideas que fundamentan este estudio es el argumento central según el cual el sexo tiene una influencia primordial en los análisis del mercado de trabajo. Pensamos que el género sigue marcando diferencias en las probabilidades de actividad, de ocupación, de trabajar por cuenta ajena, etc., es decir, que el sexo es determinante a la hora de calcular cualquiera de las probabilidades nombradas.

En el epígrafe 2 de este trabajo hacemos una revisión teórica de las contribuciones de distintos autores que analizan la influencia del género en el mercado laboral. Comenzamos el análisis 
empírico (epígrafe 3) hablando de los datos, y definiendo las variables que incorporamos en el llamado modelo de salarización. En el epígrafe 4 entramos en la explicación del modelo, en el que utilizaremos la técnica de regresión logística binaria. Expondremos los principales resultados obtenidos en el cálculo de las distintas probabilidades de trabajar por cuenta ajena, acompañándolos de algunas representaciones gráficas que avalan esas teorías. Y el epígrafe 5 lo destinamos a las conclusiones resultantes de este trabajo.

\section{Revisión de la literatura}

Según Sánchez Moreno \& Delicado Losa (2007) mientras el sexo hace referencia a desigualdades que tienen relación con la condición biológica, el concepto de género se utiliza para indicar las desigualdades tanto de carácter cultural como social asociadas a esa condición biológica. El mercado de trabajo constituye el ámbito económico y social de las desigualdades de género. En este sentido, son muchos los estudios que se han basado en las desigualdades laborales que tienen relación con el género. Es uno de los ámbitos de investigación más prolíficos en las últimas décadas, existiendo en general un acuerdo en torno a la necesidad de conocer y explicar las desigualdades existentes entre hombres y mujeres.

Existe toda una corriente literaria, desde distintas disciplinas, que establece el debate sobre el uso de los términos sexo y género. Lamas (2000) establece la categoría de género como una construcción cultural de la diferenciación sexual.

Para estudiar la segregación ocupacional que sufre la mujer, la teoría del capital humano (Becker, 1962, 1971, 1985) explica desde el punto de vista teórico el comportamiento económico de las personas, así como el papel que ejerce la formación en el mismo. Según Gradín, Otero y Arévalo (2003), la segregación constituye un problema cuando la mujer se especializa en un conjunto de ocupaciones limitado, y no lo hace fruto de una elección libre sino que se debe a condicionantes externos. Estos condicionantes pueden tener su origen en el sistema educativo, en el proceso de socialización en la familia, o en el propio mercado de trabajo, si los que emplean prefieren hombres o mujeres para determinadas actividades. Los empleadores pueden contratar a mujeres para desarrollar funciones menos técnicas, que requieran habilidades manuales, tareas parecidas a las domésticas, con menor esfuerzo físico, o actividades administrativas, entre otras. Las teorías feministas enfatizan la importancia de los estereotipos sociales de la mujer sobre los puestos de trabajo que ocupan en el mercado de trabajo. Para Del Río Otero (2003) el mercado de trabajo en Galicia, desde una perspectiva de género, refleja grandes diferencias en la colocación, presencia y retribución de hombres y mujeres. Junto a bajas tasa de actividad femenina hay altos niveles de paro, en un proceso que posiblemente esté expulsando del mercado de trabajo a muchas mujeres ante las escasas expectativas de encontrar empleo. Además, la segregación ocupacional alcanza cifras 
importantes, resultando el principal motivo de la desigualdad salarial entre hombres y mujeres.

Podemos referirnos también a la teoría del mercado de trabajo dual. Según Larrañaga Sarriegui (2000) la hipótesis básica es que el mercado de trabajo se divide en dos segmentos distintos, el sector primario o central, y el secundario o periférico. Mientras el primero ofrece puestos de trabajo con salarios elevados, posibilidades de mejora, buenas condiciones de trabajo y estabilidad en el empleo; el segundo ofrece puestos que suelen estar peor pagados, condiciones inferiores, inestabilidad y rotación entre los trabajadores. Algunos grupos sociales, como las mujeres, pueden estar integrando el mercado secundario. Esta teoría fue analizada, entre otros, por Goldin (1986) y Bulow y Summers (1986).

Muchos estudios a este respecto, señalan la persistencia de una fuerte segregación que aglutina los empleos femeninos en determinadas profesiones y ramas de actividad (muchas veces mal remunerados) y en las categorías inferiores del escalafón profesional. Para Murgatroyd (1982) existen tareas femeninas y otras no susceptibles de realizarlas los hombres.

Tanto las empresas como la sociedad en general están preparadas para cambios legislativos que eliminen obstáculos y faciliten a las mujeres el acceso a profesiones y cargos de responsabilidad para los que están sobradamente preparadas (Calvet-Puig, Lusa-Garcia, Martínez-Costa, Pons \& Tura-Solvas, 2009).

Cáceres Ruiz, Escot, Fernández y Saiz (2004) consideran que la concentración de hombres y mujeres en distintas ocupaciones y sectores constituye un hecho fundamental de la distribución del empleo. Estudian la segregación de género por ocupaciones en España, a partir de la EPA, utilizando entre otros, el índice de disimilitud (ID) de Duncan y Duncan. Para las ocupaciones, en el período 1994-2001 aumentó el ID (pasó de 0,499 a 0,5204), lo que implica que en esos años se ha producido un aumento de la segregación ocupacional de género en el mercado laboral español. También se señala que en 2001 el ID se reduce notoriamente a medida que aumenta el nivel educativo de los trabajadores.

En cuanto al papel que juega la educación en la inserción laboral, se puede afirmar que las generaciones jóvenes adquieren un nivel de formación mayor. Aunque hace años había más hombres titulados que mujeres, estas diferencias actualmente desaparecen, e incluso encontramos un porcentaje superior de mujeres en muchas titulaciones. Sin embargo, las posibilidades de acceso al mercado de trabajo así como el puesto de trabajo que ocupan es distinto, disparidades que son más evidentes entre las mujeres sin titulación.

Si el total de la población ocupada tiene 9,86 años medios de estudios, la media es de 10,52 años en las mujeres, y de 9,49 años en los hombres. Las mujeres ocupadas, por tanto, tienen 
un $10 \%$ más que los hombres en años de educación (IVIE, 2008a). En Oguiza, Allastegui y Núñez (2004), se analiza desde un punto de vista descriptivo a las mujeres ocupadas según el nivel de formación.

Podemos afirmar que el acceso a niveles educativos superiores es una forma de aumentar la participación en el empleo; además, cuando aumenta el nivel educativo en ambos géneros, el comportamiento en el mercado de trabajo de hombres y mujeres es más parecido.

En la actualidad, el nivel educativo de la población activa femenina en los tramos correspondientes a las más jóvenes es mayor que el de los hombres. Pero, este hecho no ha ido acompañado de cambios similares en el mercado de trabajo.

En cuanto a la retribución salarial, en general, cuanto más avanzada sea la edad de una mujer, menor será su salario si lo comparamos con el de un hombre que se encuentre en idénticas circunstancias laborales. En cifras del IVIE (2008b), el sueldo de una mujer mayor de 54 años es un $38 \%$ menor al de un hombre de la misma edad. El patrón de participación en la actividad en las generaciones jóvenes es mucho más parecido para hombres y mujeres que en el caso de las generaciones de más edad, donde se observan comportamientos más desiguales no sólo en el empleo, sino también en lo que respecta a los salarios.

Un tema que suscita controversia se basa en la idea de que la mujer no sólo ha de demostrar su valía, sino que en igualdad de condiciones, debe hacerlo mejor que su compañero hombre y a veces con una retribución salarial menor (Martín \& Roberts, 1984; Hartmann, 1994).

En cuanto a la diferenciación por razón de género, aunque en la actualidad se ha avanzado mucho en aras a conseguir mayores cotas de igualdad en todos los ámbitos, y concretamente en el mundo del trabajo, aún no se puede hablar de igualdad plena en cuestión de empleo entre hombres y mujeres. Además, aún existen diferencias en salarios (inferiores en muchos casos en las mujeres), en número de puestos directivos ocupados por mujeres, condiciones laborales inferiores, etc. En palabras de Barreiro García \& Martínez Seijas (2006) discriminar a la mujer en el mercado de trabajo implica discriminar a más de la mitad de la población, y por tanto, un despilfarro de capital humano.

La discriminación laboral es un factor que puede influir en la decisión de participar en el mercado de trabajo. Sáez Lara (1994) parte de la premisa de que la discriminación por razón de género en el trabajo se materializa básicamente en formas de segregación laboral, tanto horizontal (empleos propios de mujeres) como vertical (menor número en puestos de mando o responsabilidad), lo que implica diferente remuneración entre hombres y mujeres.

Al margen del papel diferenciado de la mujer en el mercado de trabajo, ha existido una preocupación creciente de la legislación de combatir la discriminación por razón del género y el trato desigual de los ciudadanos. 
Hay dos datos significativos en el proceso de la inserción femenina en el mercado de trabajo: la evolución en el empleo femenino y los cambios en la participación activa de las mujeres en el mercado laboral.

Partimos del supuesto de que la decisión de participar en el mercado de trabajo va a depender, entre otras causas, de: a) el estado civil, b) la edad $y, c)$ el nivel de estudios. Teniendo presente todas estas consideraciones, la mujer conoce el abanico de empleos a los que puede tener acceso.

En los distintos análisis de la transición de la escuela al mercado laboral, las mujeres salen al mismo ritmo que los hombres, y acceden al mercado de trabajo en la misma medida, aunque con menor éxito que ellos, ya que hay un menor porcentaje de mujeres que de hombres que realizan el paso a la ocupación, y un mayor porcentaje de ellas que lo hacen al desempleo. Los hombres tienen una mayor probabilidad de estar ocupados una vez que abandonan el sistema educativo, y menor de realizar la transición al desempleo si se compara con las mujeres (Albert, Juárez, Sánchez \& Toharia, 2003).

El incremento de la población activa en los últimos años se debe fundamentalmente a la incorporación de la mujer a la actividad. En España, la evolución de la tasa de actividad por género muestra cómo las diferencias entre las tasas en mujeres y hombres se han reducido significativamente. Por el contrario, en la ocupación esta reducción es menos notable, porque el desempleo afecta en mayor medida a las mujeres activas, con independencia de su formación. Las tasas de paro son mayores para las mujeres en todos los niveles educativos (Frutos Balibrea \& Titos Gil, 2001).

Los factores que influyen en el trabajo de las mujeres pueden ser tanto externos como internos. Son factores externos los fuertes condicionamientos de tipo familiar que tienen las mujeres en su participación laboral. Las mujeres siguen realizando una doble jornada (trabajo dentro y fuera de casa), que se traduce en desventajas para su integración plena en el mercado de trabajo.

Las dificultades para hacer compatible el trabajo en casa con el trabajo remunerado, pueden llevar a un abandono transitorio del trabajo, en períodos de inactividad forzosa. Este ciclo laboral discontinuo de las mujeres, que obstaculiza las posibilidades de desarrollo profesional, tiende a ser menor en las generaciones más jóvenes. Este hecho hay que acompañarlo de unas cifras de natalidad bajas. Los factores internos se ubican básicamente en el ámbito cultural.

Ante la dispar situación de las mujeres en el mercado laboral, la opción de trabajar por cuenta propia puede resultar interesante, ya que se trata de una modalidad de trabajo que puede generar empleo. Esta modalidad del trabajo autónomo, además de favorecer la creación de 
empleo, puede también contribuir al desarrollo económico. En las Jornadas de emprendizaje de la Asociación Mujeres y Tecnología (2007) se analizó el autoempleo como alternativa de inserción laboral.

Si hasta hace poco las empresas de mujeres quedaban relegadas en el mundo empresarial, hoy en día la alta cualificación de la población activa femenina hace esperar una actividad empresarial dirigida por mujeres, con mayores metas y orientada a actividades destacadas en el entorno productivo. En este sentido, Guzmán Cuevas \& Rodríguez Gutiérrez (2008) consideran que las mujeres adoptan un comportamiento diferente al de los hombres en el desempeño de sus labores empresariales. Rosener (1990) se refiere al comportamiento diferente de las mujeres en el desempeño de sus funciones en la gerencia empresarial. En las investigaciones de Gatewood, Shaver y Gartner (1995) y Buttner y Moore (1997) se concluye que las mujeres basan su éxito empresarial no sólo en criterios económicos, sino que está más relacionado con las motivaciones que les llevaron a crear una empresa, como la autorrealización y el propio desarrollo profesional.

Freire Seoane y Teijeiro Alvarez (2009) señalan que en el contexto actual, el perfil del emprendedor europeo medio es el siguiente: hombre, de aproximadamente 35 años, que previamente trabajó en una empresa pequeña, en un puesto de trabajo de dirección o como trabajador cualificado, y con un nivel de formación y experiencia medio. Las motivaciones que le han llevado a crear su propia empresa fueron básicamente, la autorrealización, el deseo de independencia y el autoempleo.

En el estudio de Riobóo Lestón y Martín López (2011) se mide el nivel de igualdad o desigualdad existente en el mercado laboral en Galicia y España mediante la obtención de un índice sintético (Gender Inequality in Labour Market, GILM) que permite cuantificar las diferencias entre géneros; en esa construcción, las variables se desagregan en función del género.

Con respecto a la aportación de este trabajo, igualmente las variables se desagregan por género, pero con otra finalidad. La diferencia que este trabajo de investigación supone frente a la literatura publicada hasta el momento, es que nos interesa conocer las probabilidades de que una persona trabaje por cuenta ajena bajo determinadas premisas. La variable explicada es dicotómica y el género forma parte de la definición de las variables explicativas. La forma de construir una de esas variables, la llamada Ingresos del hogar corregidos es novedosa, y por tanto, original. 


\section{Análisis empírico. Definición de variables}

Nos interesa el estudio de las probabilidades de trabajar por cuenta ajena o por cuenta propia. Introduciremos variables tanto cualitativas como cuantitativas. Una característica importante es que todas las variables sean categóricas o cuantitativas las vamos a generar en su interacción con el sexo.

Comprobaremos que la interacción de la variable sexo con las demás variables explicativas es esencial para comprender el papel de los distintos factores que influyen en las decisiones de los individuos.

El modelo lo llamaremos modelo de salarización. Aplicaremos la regresión logística binaria (Agresti, 1996; Amemiya, 1981; Cox \& Snell, 1989; Hosmer \& Lemeshow, 1989) tanto para el estudio estadístico de los distintos resultados, como para representar de un modo gráfico las distintas probabilidades bajo determinadas condiciones.

Al entrar en el estudio del modelo nos interesa conocer las razones, traducidas en términos de variables, que conducen a una persona a trabajar por cuenta ajena o por cuenta propia.

\section{Datos}

En nuestra investigación vamos a utilizar microdatos extraídos de la "Encuesta de condiciones de vida de las familias" que fueron facilitados por el Instituto Galego de Estatística (IGE) y que corresponden al año 2003. La metodología utilizada para la elaboración de la Encuesta fue realizada por el IGE (2005). La "Enquisa de condicións de vida das familias", o en castellano, "Encuesta de condiciones de vida de las familias" (ECV) es una actividad estadística con periodicidad anual que realiza el IGE desde 1999. Se trata de una encuesta destinada a los hogares gallegos con la finalidad de obtener información sobre sus características socioeconómicas. Se enmarca dentro del ámbito de información referente al bienestar social.

Para la elaboración de la Encuesta participaron 18.572 personas que constituyen el tamaño de la muestra, y representan a una población de 2.701.425 personas, total de residentes en Galicia en ese año. Aunque para este trabajo de investigación hemos partido de la ECV, hemos comprobado que hay diferencias en lo que se refiere a la población gallega total con los datos publicados en el Padrón Municipal de habitantes. En el Padrón, el total poblacional es de 2.751.094.

La población apenas ha variado en estos últimos años. El último dato de población de la Comunidad gallega publicado por el INE (Padrón Municipal de Habitantes) en 2013 con fecha de referencia 1 de enero es de 2.763.499 personas.

La muestra de la ECV, al ser una muestra no autoponderada, para cualquier estimación que se quiera realizar con los datos, se les deberá aplicar el factor de elevación de cada registro, la 
variable "frep", y que permite extrapolar los datos de la encuesta para el total de la población. De este modo, en el caso de la estimación del total de una característica $X$ ésta será la suma del total de registros que presenten la misma (Xi) multiplicados por sus correspondientes factores de ponderación; $\quad \hat{X}=\sum X i *$ frep $i$

En el fichero hay tantas filas como personas en la muestra. A cada persona le corresponde un factor de elevación, de tal manera que todas las personas de una vivienda tienen ese mismo factor de elevación. La suma de todos los factores de elevación es justamente el total de la población gallega.

Como todo nuestro interés se centra en personas que de una u otra forma pertenecen al mercado de trabajo, o al menos por su edad pueden tener algún tipo de vinculación con él, hemos comenzado por separar al colectivo de 16-65 años, que hasta el año de elaboración de este trabajo, fueron los años de inicio y fin de la vida laboral de una persona. La muestra inicial y la población a la que representa los hemos recogido en las tablas 1 y 2 .

\begin{tabular}{|c|c|c|c|}
\hline & & & Cuenta ajena: \\
& & Ocupados: & 4906 personas \\
\cline { 3 - 3 } Muestra de & Activos: & 6836 personas & Cuenta propia: \\
16-65 años & 7555 personas & & 1930 personas \\
\cline { 3 - 4 }$(\mathbf{1 1 9 0 0}$ personas) & & Parados: & \\
\cline { 2 - 4 } & & 719 personas & \\
\cline { 2 - 4 } & Inactivos: & & \\
& 4345 personas & & \\
\end{tabular}

Tabla 1. Número de personas que forman parte de la muestra. Elaboración propia a partir de IGE (2005), "Enquisa de condicións de vida das familias", año 2003, Metodología 2005

\begin{tabular}{|c|c|c|c|}
\hline \multirow{4}{*}{$\begin{array}{c}\text { Población de } \\
\text { 16-65 años } \\
\text { (1814894 } \\
\text { personas) }\end{array}$} & \multirow{3}{*}{$\begin{array}{c}\text { Activos: } \\
1183240 \text { personas }\end{array}$} & \multirow{2}{*}{$\begin{array}{c}\text { Ocupados: } \\
1063801 \text { personas }\end{array}$} & $\begin{array}{c}\text { Cuenta ajena: } \\
784099 \text { personas }\end{array}$ \\
\hline & & & $\begin{array}{l}\text { Cuenta propia: } \\
279702 \text { personas }\end{array}$ \\
\hline & & $\begin{array}{c}\text { Parados: } \\
119440 \text { personas }\end{array}$ & \\
\hline & $\begin{array}{c}\text { Inactivos: } \\
631654 \text { personas }\end{array}$ & & \\
\hline
\end{tabular}

Tabla 2. Número de personas que forman parte de la población. Elaboración propia a partir de IGE (2005), "Enquisa de condicións de vida das familias", año 2003, Metodología 2005

En este modelo aplicaremos la regresión logística binaria partiendo del colectivo de ocupados. Queremos analizar dentro de las personas que trabajan, las razones que hacen que sean o bien trabajadores por cuenta ajena o bien por cuenta propia. En la muestra, el número de personas ocupadas lo forman 6.836 , de las cuales 4.906 son trabajadores por cuenta ajena y 1.930 son trabajadores por cuenta propia. En términos de población, se traduce en 1.063 .801 personas ocupadas, de las cuales 784.099 son trabajadores por cuenta ajena y 279.702 son trabajadores por cuenta propia. 
El método es, por consiguiente, la regresión logística binaria y el programa informático utilizado es el SPSS.

\section{Variables utilizadas}

En este epígrafe reflejaremos las variables extraídas de la ECV y otras que hemos creado a partir de los datos pertenecientes a la ECV. Trataremos de averiguar cuáles pueden ser los condicionantes que hacen que una persona trabaje por cuenta ajena o por cuenta propia.

En el modelo de salarización, la variable dependiente la llamamos "tipo de empleador" con código 0 si son trabajadores por cuenta propia y 1 si son trabajadores por cuenta ajena. La clasificación la haremos a partir de la variable "sitprof" (situación profesional en el trabajo actual) obtenida de la ECV. Son trabajadores por cuenta propia los siguientes:

- Empresario o profesional sin asalariados

- Ayuda familiar

- Miembro de cooperativa

- Empresario o profesional con 10 o más asalariados

- Empresario o profesional con menos de 10 asalariados

y son trabajadores por cuenta ajena:

- Asalariado

- Trabajador en formación

- Aprendiz remunerado.

La fundamentación de las definiciones de las variables independientes consiste en afirmar que el trabajo según el tipo de empleador no es igual en los hombres que en las mujeres y, como consecuencia, el sexo es una variable con un papel especial en los modelos del mercado de trabajo. Además, hay estudios que afirman que hay importantes diferencias entre hombres y mujeres respecto a la influencia de otras variables sociodemográficas, corroborando destacadas discrepancias entre ambos sexos en las contribuciones de otras características personales como el estado civil, el nivel educativo, la edad, etc.

En el modelo utilizaremos como variables independientes 7 variables categóricas y 2 variables cuantitativas. El tratamiento que le damos a las variables categóricas es distinto que a las variables cuantitativas o numéricas.

Las variables categóricas son: sexo, grupos de edad, estado civil, estudios realizados, nacionalidad, tamaño del municipio y sector. 
Dada la importancia de la interacción entre el sexo y las demás variables explicativas, los modelos iniciales incluyen las siguientes variables categóricas:

- Sexo

- Grupos de edad*sexo

- Estado civil*sexo

- Estudios realizados*sexo

- Nacionalidad*sexo

- Tamaño municipio*sexo

- Sector*sexo

Las distintas variables categóricas consideradas tienen las modalidades o categorías que se recogen en la tabla 3.

Con respecto a la variable "Estudios realizados", la clasificación que hemos utilizado la hemos obtenido mediante agrupaciones de la clasificación CNED, que es la que aplica el IGE.

Las variables cuantitativas, al igual que las variables categóricas, también tienen en cuenta la relación entre su efecto y el sexo de la persona analizada. Esta interacción se refleja en la construcción de las variables categóricas duplicando los códigos en la misma variable (en función del sexo), mientras que en las variables cuantitativas se crea una variable para cada sexo. Así, en nuestros modelos, las variables cuantitativas son:

- Número miembros*sexo

- Ingresos del hogar corregidos*sexo

La variable "Número de miembros" es el número de personas que integran la unidad familiar. Esta variable toma un valor mínimo de 1 y un valor máximo de 8.

Para la explicación de la variable "Ingresos del hogar corregidos" necesitamos hacer alusión a las variables de ingresos que se encuentran en el fichero de datos de la ECV. Las unidades de los ingresos son euros al mes. La variable ingresos totales de las personas es la suma de ingresos por trabajo por cuenta ajena, ingresos por trabajo por cuenta propia, ingresos por prestaciones, ingresos de prestaciones o subsidios por desempleo, ingresos de rentas e ingresos por otros ingresos. 


\begin{tabular}{|c|c|}
\hline Atributos & Modalidades \\
\hline \multirow{2}{*}{ Sexo } & Hombre \\
\hline & Mujer \\
\hline \multirow{5}{*}{ Estado civil } & Soltero \\
\hline & Casado \\
\hline & Separado \\
\hline & Divorciado \\
\hline & Viudo \\
\hline \multirow{10}{*}{$\begin{array}{l}\text { Grupos de edad } \\
\text { (de } 5 \text { en } 5 \text { años) }\end{array}$} & De 16 a 20 años \\
\hline & De 21 a 25 años \\
\hline & De 26 a 30 años \\
\hline & De 31 a 35 años \\
\hline & De 36 a 40 años \\
\hline & De 41 a 45 años \\
\hline & De 46 a 50 años \\
\hline & De 51 a 55 años \\
\hline & De 56 a 60 años \\
\hline & De 61 a 65 años \\
\hline \multirow{11}{*}{ Estudios realizados } & Analfabetos \\
\hline & Estudios primarios incompletos \\
\hline & Estudios primarios completos \\
\hline & Estudios sin graduado escolar \\
\hline & Estudios con graduado escolar \\
\hline & Bachillerato y similar \\
\hline & Grado medio formación profesional \\
\hline & Grado superior formación profesional \\
\hline & Primer ciclo universidad \\
\hline & Especialización profesional \\
\hline & Segundo ciclo universidad y doctorado \\
\hline \multirow{2}{*}{ Nacionalidad } & Española y doble nacionalidad \\
\hline & Extranjera \\
\hline \multirow{3}{*}{$\begin{array}{l}\text { Tamaño } \\
\text { municipio }\end{array}$} & Menos de 10.000 habitantes \\
\hline & Entre 10.000 y 20.000 habitantes \\
\hline & Más de 20.000 habitantes \\
\hline \multirow{7}{*}{ Sector } & Agricultura \\
\hline & Pesca \\
\hline & Energía \\
\hline & Industria \\
\hline & Construcción \\
\hline & Servicios en general \\
\hline & Administración Pública, Defensa y S.Social obligatoria \\
\hline
\end{tabular}

Tabla 3. Variables categóricas y sus correspondientes modalidades. Elaboración propia a partir de IGE (2005), "Enquisa de condicións de vida das familias", año 2003, Metodología 2005.

Una de las aportaciones novedosas de este trabajo es la creación y la forma de construir esta variable. Hemos establecido el siguiente planteamiento: en la decisión de un individuo de participar en el mercado de trabajo, y en qué condiciones lo va a hacer, todos los ingresos del hogar son relevantes salvo los generados por la relación con el mercado de trabajo de la persona que estemos analizando en cada momento. En base a este razonamiento, definimos los ingresos del hogar corregidos, que es el importe total de los ingresos del hogar descontando los ingresos por trabajo, ya sea por cuenta ajena o por cuenta propia, y de prestaciones o subsidios por desempleo de la persona considerada. Sin embargo, se incluyen 
todos los ingresos por prestaciones, los ingresos de rentas, y otros ingresos, con independencia de la persona que los reciba.

Por tanto, mide los ingresos que recibe el hogar que no dependen de lo que aporta la persona analizada por su relación, sea cual sea ésta, con el mercado de trabajo. De este modo, cuanto mayor sean los ingresos del hogar corregidos, menos necesidad tiene el individuo de trabajar, ya que en principio las necesidades básicas estarían cubiertas con otras rentas familiares. Por construcción, los ingresos del hogar corregidos no son iguales para todos los miembros del hogar; cuanto mayores sean los ingresos que aporte un miembro concreto por rentas del trabajo o subsidios por desempleo, menor será su ingreso del hogar corregido, y mayores los ingresos del hogar corregidos de los demás miembros.

\section{Modelo de salarización}

En este epígrafe analizaremos en primer lugar la importancia del sector de actividad en el modelo. Posteriormente, explicaremos la metodología empleada; ésta se dividirá en dos partes:

- En una primera parte, expondremos distintos modelos de regresión logística binaria, o modelos logit. En el análisis de cada uno de los modelos veremos cómo elegimos el mejor desde un punto de vista estadístico.

- En una segunda parte, y a partir del mejor modelo plantearemos los parámetros resultantes de la aplicación logit, tabla de clasificación, y cálculo de las distintas probabilidades bajo determinados supuestos.

El objetivo del modelo es estudiar la probabilidad de trabajar por cuenta ajena/cuenta propia a partir de las características personales del individuo.

\section{La importancia del sector de actividad}

Si nos centramos en el colectivo de las personas que trabajan, el sector de actividad es una variable básica para estimar la probabilidad de que el trabajador lo haga por cuenta propia o por cuenta ajena. Es bien sabido que el peso relativo de cada colectivo varía mucho con el sector que se considere, de modo que la bondad del ajuste mejorará de forma sustancial si incorporamos esta información al modelo.

En esta variable partimos de la clasificación CNAE que utiliza el IGE, de forma que esas actividades las agrupamos en siete grandes ramas (véase tabla 3 ).

Al incluir el sector como una variable explicativa, nos encontramos con el problema de que no podemos trabajar con todos los ocupados. La razón está en que en los grupos 3 y 7 de la variable sector, que corresponden a Energía y Administración Pública, no hay trabajadores por cuenta propia. Por este motivo, excluimos del estudio a estos dos grupos. Por ello, la muestra 
la forman 6.363 personas que representan a 986.796 personas en el colectivo total (véase tabla 4).

\begin{tabular}{|l|c|r|}
\hline \multicolumn{1}{|c|}{ Modelo } & $\begin{array}{c}\text { Tamaño } \\
\text { muestra }\end{array}$ & \multicolumn{1}{|c|}{$\begin{array}{c}\text { Tamaño } \\
\text { población }\end{array}$} \\
\hline Todos los sectores de actividad & 6.836 & 1.063 .801 \\
\hline Excluyendo sector Energía y Admón. Pública & 6.363 & 986.796 \\
\hline
\end{tabular}

Tabla 4. Tamaño poblacional y muestral inicial y final (excluyendo dos sectores). Elaboración propia a partir de IGE (2005), "Enquisa de condicións de vida das familias", año 2003, Metodología 2005

\section{Tipo de empleador: el modelo estadístico}

Se ha utilizado la metodología de regresión logística binaria porque es adecuada cuando la variable dependiente es dicotómica y porque nos interesa el cálculo de las probabilidades estableciendo determinadas condiciones.

En el modelo de salarización, planteamos la siguiente hipótesis: el modelo en el que todas las variables interactúan con el sexo (modelo general) es el que arroja mejores resultados. Con el fin de comprobar si nuestra idea inicial es o no cierta, y para la elección del mejor modelo se estiman distintas versiones, en cada una se siguen estos pasos:

- Se escoge una de las variables independientes sin interactuar con el sexo, manteniendo las demás variables desglosadas.

- Se estima este modelo (modelo restringido).

- Repetimos este proceso tantas veces como variables tengamos.

- Comparamos estos hipotéticos modelos con el modelo en que todas las variables interactúan con el sexo (modelo general).

La elección del modelo más adecuado se realiza mediante el contraste de razón de verosimilitud:

$$
-2 \ln L_{r}-\left(-2 \ln L_{g}\right) \rightarrow \chi^{2} n-m
$$

donde $L$ denota el valor de la verosimilitud, $r$ se refiere al modelo restringido, $g$ se refiere al modelo general, $\mathrm{n}$ es el número de parámetros del modelo general, $\mathrm{m}$ es el número de parámetros del modelo restringido, $\chi_{n-m}^{2} y$ es la distribución chi-cuadrado con $n-m$ grados de libertad.

Se estimaron distintas versiones: el modelo general, y varios modelos restringidos. En el modelo general, el sexo interactúa con todas las demás variables explicativas. En cada especificación restringida, se fuerza a que el efecto de una variable explicativa sea el mismo para hombres y mujeres, en tanto que se mantiene la interacción en las demás. Dado que hay ocho variables explicativas además del sexo, esto hace un total de ocho modelos restringidos. 
En la tabla 5 recogemos los resultados obtenidos en estas estimaciones. Añadimos en la última fila los resultados correspondientes al modelo general, que vamos a necesitar para hacer la comparativa con los otros modelos.

\begin{tabular}{|c|c|c|c|c|c|c|}
\hline $\begin{array}{c}\text { VARIABLE } \\
\text { RESTRINGIDA }\end{array}$ & $\mathbf{R}^{2}$ Nagelkerke & $\begin{array}{c}\text { No total } \\
\text { parámetros } \\
\text { estimados } \\
\text { (con la } \\
\text { constante) }\end{array}$ & $\begin{array}{c}\% \\
\text { correctamente } \\
\text { clasificados }\end{array}$ & \begin{tabular}{|c|}
$\quad N^{\circ}$ \\
restricciones \\
respecto al \\
$\quad$ modelo \\
general
\end{tabular} & $\begin{array}{l}\text { Valor del estadístico } \\
-2 \ln L_{r}-\left(-2 \ln L_{g}\right)\end{array}$ & p-valor \\
\hline Grupos de edad & 0,357 & 57 & 79,8 & 9 & 2683,344 & $<0,01$ \\
\hline Estado civil & 0,357 & 62 & 79,8 & 4 & 2330,47 & $<0,01$ \\
\hline Nacionalidad & 0,360 & 65 & 79,9 & 1 & 233,266 & $<0,01$ \\
\hline \begin{tabular}{|l} 
Estudios \\
realizados
\end{tabular} & 0,355 & 56 & 79,8 & 10 & 4290,583 & $<0,01$ \\
\hline Sector & 0,355 & 62 & 79,8 & 4 & 4875,6 & $<0,01$ \\
\hline $\begin{array}{l}\text { Tamaño } \\
\text { municipio }\end{array}$ & 0,360 & 64 & 79,8 & 2 & 308,426 & $<0,01$ \\
\hline $\begin{array}{l}\text { Número } \\
\text { miembros }\end{array}$ & 0,360 & 65 & 79,9 & 1 & 21,861 & $<0,01$ \\
\hline $\begin{array}{l}\text { Ingresos del } \\
\text { hogar } \\
\text { corregidos }\end{array}$ & 0,360 & 65 & 79,9 & 1 & 50,392 & $<0,01$ \\
\hline $\begin{array}{l}\text { MODELO } \\
\text { GENERAL }\end{array}$ & 0,360 & 66 & 79,9 & & & \\
\hline
\end{tabular}

Tabla 5. Principales resultados en los modelos restringidos y en el modelo general. Contraste de la validez de las restricciones que los definen. Elaboración propia a partir de IGE (2005),

"Enquisa de condicións de vida das familias", año 2003, Metodología 2005

En todos los casos rechazamos la hipótesis nula, lo que significa que el mejor modelo es el general y es el que vamos a considerar en el estudio logit posterior.

Seguidamente exponemos en la tabla 6 los resultados de la estimación logit del modelo de salarización, que hemos considerado mejor por las razones anteriores.

Con respecto a los coeficientes, comprobamos que todos son significativos al $5 \%$ con la excepción de número miembros hombre.

\begin{tabular}{|l|r|r|r|r|r|}
\hline \multicolumn{7}{|c|}{ Variables } & \multicolumn{1}{|c|}{ B } & E.T. & Wald & Sig. & Exp(B) \\
\hline \multicolumn{7}{|c|}{ Gexo } \\
\hline Hombre & 2,293 & 0,132 & 299,572 & 0,000 & 9,907 \\
\hline Hombres de 16 a 20 años & 2,219 & 0,032 & 4697,760 & 0,000 & 9,199 \\
\hline Hombres de 21 a 25 años & 1,842 & 0,024 & 5694,837 & 0,000 & 6,312 \\
\hline Hombres de 26 a 30 años & 1,150 & 0,021 & 2938,925 & 0,000 & 3,158 \\
\hline Hombres de 31 a 35 años & 1,172 & 0,020 & 3507,817 & 0,000 & 3,230 \\
\hline Hombres de 36 a 40 años & 0,733 & 0,019 & 1458,020 & 0,000 & 2,080 \\
\hline Hombres de 41 a 45 años & 0,853 & 0,019 & 1985,880 & 0,000 & 2,347 \\
\hline Hombres de 46 a 50 años & 0,689 & 0,019 & 1271,997 & 0,000 & 1,991 \\
\hline Hombres de 51 a 55 años & 0,484 & 0,019 & 644,312 & 0,000 & 1,622 \\
\hline Hombres de 56 a 60 años & 0,139 & 0,020 & 48,430 & 0,000 & 1,149 \\
\hline
\end{tabular}




\begin{tabular}{|c|c|c|c|c|c|}
\hline Variables & B & E.T. & Wald & Sig. & $\operatorname{Exp}(B)$ \\
\hline Mujeres de 16 a 20 años & 0,825 & 0,040 & 422,726 & 0,000 & 2,282 \\
\hline Mujeres de 21 a 25 años & 1,809 & 0,032 & 3207,478 & 0,000 & 6,106 \\
\hline Mujeres de 26 a 30 años & 1,524 & 0,028 & 2923,619 & 0,000 & 4,590 \\
\hline Mujeres de 31 a 35 años & 1,026 & 0,027 & 1484,475 & 0,000 & 2,790 \\
\hline Mujeres de 36 a 40 años & 1,241 & 0,026 & 2197,967 & 0,000 & 3,460 \\
\hline Mujeres de 41 a 45 años & 0,865 & 0,026 & 1109,086 & 0,000 & 2,374 \\
\hline Mujeres de 46 a 50 años & 0,774 & 0,026 & 878,723 & 0,000 & 2,168 \\
\hline Mujeres de 51 a 55 años & 0,439 & 0,026 & 286,457 & 0,000 & 1,551 \\
\hline Mujeres de 56 a 60 años & 0,144 & 0,027 & 28,477 & 0,000 & 1,155 \\
\hline \multicolumn{6}{|c|}{ Estado civil*sexo } \\
\hline Hombres solteros & 1,170 & 0,036 & 1046,761 & 0,000 & 3,221 \\
\hline Hombres casados & 0,871 & 0,035 & 618,679 & 0,000 & 2,389 \\
\hline Hombres separados & 0,971 & 0,042 & 529,818 & 0,000 & 2,641 \\
\hline Hombres divorciados & 1,136 & 0,049 & 531,124 & 0,000 & 3,115 \\
\hline Mujeres solteras & $-0,060$ & 0,028 & 4,667 & 0,031 & 0,942 \\
\hline Mujeres casadas & $-0,825$ & 0,025 & 1093,263 & 0,000 & 0,438 \\
\hline Mujeres separadas & $-0,399$ & 0,033 & 147,852 & 0,000 & 0,671 \\
\hline Mujeres divorciadas & $-0,521$ & 0,042 & 157,335 & 0,000 & 0,594 \\
\hline \multicolumn{6}{|c|}{ Nacionalidad*sexo } \\
\hline Hombre español y de doble nacionalidad & $-0,266$ & 0,031 & 75,074 & 0,000 & 0,766 \\
\hline Mujer española y de doble nacionalidad & 0,420 & 0,033 & 163,674 & 0,000 & 1,521 \\
\hline \multicolumn{6}{|c|}{ Estudios realizados*sexo } \\
\hline $\begin{array}{l}\text { Hombres con estudios primarios } \\
\text { incompletos }\end{array}$ & $-2,392$ & 0,106 & 512,239 & 0,000 & 0,091 \\
\hline Hombres con estudios primarios completos & $-3,289$ & 0,105 & 978,994 & 0,000 & 0,037 \\
\hline Hombres sin graduado escolar & $-2,005$ & 0,103 & 377,135 & 0,000 & 0,135 \\
\hline Hombres con graduado escolar & $-2,304$ & 0,103 & 499,880 & 0,000 & 0,100 \\
\hline Hombres con bachillerato y similar & $-2,294$ & 0,104 & 491,192 & 0,000 & 0,101 \\
\hline $\begin{array}{l}\text { Hombres con grado medio formación } \\
\text { profesional }\end{array}$ & $-1,904$ & 0,104 & 333,226 & 0,000 & 0,149 \\
\hline $\begin{array}{l}\text { Hombres con grado superior formación } \\
\text { profesional }\end{array}$ & $-1,968$ & 0,104 & 360,500 & 0,000 & 0,140 \\
\hline Hombres con primer ciclo universidad & $-1,968$ & 0,104 & 357,125 & 0,000 & 0,140 \\
\hline Hombres con especialización profesional & $-1,372$ & 0,108 & 162,305 & 0,000 & 0,254 \\
\hline $\begin{array}{l}\text { Hombres segundo ciclo universidad y } \\
\text { doctorado }\end{array}$ & $-2,167$ & 0,104 & 434,966 & 0,000 & 0,115 \\
\hline Mujeres con estudios primarios incompletos & 0,520 & 0,068 & 59,078 & 0,000 & 1,683 \\
\hline Mujeres con estudios primarios completos & 0,624 & 0,069 & 82,077 & 0,000 & 1,866 \\
\hline Mujeres sin graduado escolar & 0,775 & 0,062 & 154,716 & 0,000 & 2,171 \\
\hline Mujeres con graduado escolar & 1,086 & 0,062 & 308,559 & 0,000 & 2,963 \\
\hline Mujeres con bachillerato y similar & 0,985 & 0,062 & 249,522 & 0,000 & 2,678 \\
\hline $\begin{array}{l}\text { Mujeres con grado medio formación } \\
\text { profesional }\end{array}$ & 1,798 & 0,064 & 777,184 & 0,000 & 6,035 \\
\hline $\begin{array}{l}\text { Mujeres con grado superior formación } \\
\text { profesional }\end{array}$ & 0 & 0 & 175 & 0,000 & ,903 \\
\hline Mujeres con primer ciclo universidad & 2,283 & 0,064 & 1276,806 & 0,000 & 9,803 \\
\hline Mujeres con especialización profesional & 2,316 & 0,071 & 1068,215 & 0,000 & 10,132 \\
\hline $\begin{array}{l}\text { Mujeres segundo ciclo universidad y } \\
\text { doctorado }\end{array}$ & 1,285 & 0,063 & 410,683 & 0,000 & 3,614 \\
\hline
\end{tabular}




\begin{tabular}{|c|c|c|c|c|c|}
\hline Variables & B & E.T. & Wald & Sig. & $\operatorname{Exp}(B)$ \\
\hline \multicolumn{6}{|c|}{ Tamaño municipio*sexo } \\
\hline $\begin{array}{l}\text { Hombres municipios de menos de } 10000 \\
\text { habitantes }\end{array}$ & $-0,579$ & 0,008 & 5373,831 & 0,000 & 0,561 \\
\hline $\begin{array}{l}\text { Hombres municipios entre } 10000-20000 \\
\text { habitantes }\end{array}$ & $-0,486$ & 0,012 & 1747,181 & 0,000 & 0,615 \\
\hline $\begin{array}{l}\text { Mujeres municipios de menos de } 10000 \\
\text { habitantes }\end{array}$ & $-0,794$ & 0,010 & 6116,347 & 0,000 & 0,452 \\
\hline $\begin{array}{l}\text { Mujeres municipios entre } 10000-20000 \\
\text { habitantes }\end{array}$ & $-0,536$ & 0,015 & 1228,544 & 0,000 & 0,585 \\
\hline \multicolumn{6}{|c|}{ Sector*sexo } \\
\hline Hombres agricultura & $-2,271$ & 0,014 & 25255,068 & 0,000 & 0,103 \\
\hline Hombres pesca & 0,077 & 0,016 & 21,628 & 0,000 & 1,080 \\
\hline Hombres industria & 0,955 & 0,010 & 9743,122 & 0,000 & 2,598 \\
\hline Hombres construcción & 0,445 & 0,009 & 2351,808 & 0,000 & 1,561 \\
\hline Mujeres agricultura & $-3,610$ & 0,025 & 21065,583 & 0,000 & 0,027 \\
\hline Mujeres pesca & $-1,696$ & 0,032 & 2792,828 & 0,000 & 0,183 \\
\hline Mujeres industria & 0,901 & 0,014 & 4377,969 & 0,000 & 2,462 \\
\hline Mujeres construcción & 0,367 & 0,046 & 64,962 & 0,000 & 1,444 \\
\hline \multicolumn{6}{|c|}{ No miembros*sexo } \\
\hline No miembros hombre & 0,001 & 0,004 & 0,022 & 0,883 & 1,001 \\
\hline No miembros mujer & $-0,026$ & 0,005 & 33,843 & 0,000 & 0,974 \\
\hline \multicolumn{6}{|c|}{ Ingresos del hogar corregidos* sexo } \\
\hline Ingresos hombre & $-0,003$ & 0,000 & 237,802 & 0,000 & 0,997 \\
\hline Ingresos mujer & $-0,001$ & 0,000 & 9,581 & 0,002 & 0,999 \\
\hline Constante & $-0,308$ & 0,068 & 20,265 & 0,000 & 0,735 \\
\hline Observaciones con variable dependiente $=0$ & 1930 & & & & \\
\hline Observaciones con variable dependiente $=1$ & 4433 & & & & \\
\hline Total de observaciones & 6363 & & & & \\
\hline Chi-cuadrado & $\begin{array}{r}284862,14 \\
9\end{array}$ & & $p=0,000$ & & \\
\hline-2 log de la verosimilitud & $\begin{array}{r}891747,50 \\
7\end{array}$ & & & & \\
\hline $\mathrm{R}^{2}$ de Nagelkerke & 0,360 & & & & \\
\hline $\mathrm{R}^{2}$ de MacFadden & 0,242 & & & & \\
\hline Predicciones correctas & $79,9 \%$ & & & & \\
\hline
\end{tabular}

NOTA: La categoría de referencia es mujer, de 61-65 años, viuda, extranjera, analfabeta, que pertenece a un municipio de más de 20000 habitantes y que trabaja en el sector servicios.

Tabla 6. Resultados de la estimación logit del modelo de salarización. Variable dependiente: TIPO DE EMPLEADOR, Método: Logit binario estimado por máxima verosimilitud, Muestra: 6363 observaciones.

Elaboración propia a partir de IGE (2005), "Enquisa de condicións de vida das familias", año 2003,

Metodología 2005 
La tabla 7 recoge los porcentajes de trabajadores correctamente clasificados según el modelo estimado.

\begin{tabular}{|c|c|c|c|c|}
\hline \multirow{2}{*}{$\begin{array}{c}\text { Población analizada: } \\
\text { Personas ocupadas }\end{array}$} & \multicolumn{2}{c|}{ Pronosticado } & \multirow{2}{*}{$\begin{array}{c}\text { Porcentaje } \\
\text { correcto }\end{array}$} \\
\cline { 3 - 4 } & $\begin{array}{c}\text { Cuenta } \\
\text { propia }\end{array}$ & $\begin{array}{c}\text { Cuenta } \\
\text { ajena }\end{array}$ & 38,7 \\
\hline \multirow{2}{*}{ Observado } & Cuenta propia & 108298 & 171404 & 96,2 \\
\cline { 2 - 4 } & Cuenta ajena & 27042 & 680052 & 79,9 \\
\hline \multicolumn{2}{|c|}{ Porcentaje global } & & & \\
\hline
\end{tabular}

Nota: El valor de corte es 0,500

Tabla 7. Clasificación de los individuos según el modelo estimado de salarización. Elaboración propia a partir de IGE (2005), "Enquisa de condicións de vida das familias", año 2003, Metodología 2005

Comparar el número de casos observados con los pronosticados por el modelo estimado nos permite evaluar la idoneidad del modelo. En el análisis de este modelo, la capacidad predictiva, medida por el porcentaje de aciertos, es del 79,9\% (valor de corte 0,5).

La "sensibilidad del modelo" (proporción de trabajadores por cuenta ajena clasificados correctamente) es muy elevada, concretamente 96,2\%. Sin embargo, la "especificidad" del mismo (proporción de trabajadores por cuenta propia clasificados correctamente) es del $38,7 \%$.

Una vez conocidos los coeficientes resultantes de la estimación logit, nuestro siguiente objetivo es determinar la probabilidad de trabajar por cuenta ajena. Por ello, expondremos en términos generales, cómo calcular estas probabilidades en la línea de otras publicaciones que utilizan la misma metodología (con diferencias que se refieren al cálculo de las probabilidades en el caso de las variables cuantitativas).

Clasificaremos la población en tres colectivos de interés: personas entre 21 y 35 años (jóvenes), entre 36 y 50 años (mediana edad) y entre 51 y 65 años (mayores). Dentro de cada uno de estos grupos haremos tres subgrupos que dividen las edades en intervalos de amplitud fija, concretamente cada 5 años.

Al analizar la influencia de cada una de las variables categóricas, consideraremos fijas 2 de esas variables: nacionalidad y tamaño del municipio. Partiremos de españoles que pertenecen a municipios de más de 20.000 habitantes (municipios grandes). La nacionalidad y el tamaño del municipio las tomamos fijas. Sin embargo, el sexo, el estado civil, los estudios realizados y la edad son variables. 
Para el cálculo de las distintas probabilidades sustituimos el valor de los coeficientes obtenidos en la tabla 6 en la formulación del modelo logit siguiente:

$$
p=\frac{1}{1+e^{-\left(\beta_{0}+\beta_{1} X_{1}+\ldots+\beta_{k} X_{k}\right)}}
$$

con la excepción de que en las variables cuantitativas cada coeficiente lo vamos a multiplicar por la media correspondiente, es decir:

Parámetro Ingresos corregidos hombre/mujer*renta media ingresos corregidos hombre/mujer Parámetro Número miembros hombre/mujer*número medio miembros hombre/mujer.

En la tabla 8 aparecen recogidos los valores medios que, para las distintas variables son: 1,77, $1,18,672,99$ y 611,54 .

\begin{tabular}{|l|r|r|r|r|}
\cline { 2 - 5 } \multicolumn{1}{c|}{} & Mínimo & \multicolumn{1}{c|}{ Máximo } & \multicolumn{1}{c|}{ Media } & \multicolumn{1}{c|}{$\begin{array}{c}\text { Desviación } \\
\text { típica }\end{array}$} \\
\hline No miembros hombre & 1 & 8 & 1,77 & 1,730 \\
\hline No miembros mujer & 1 & 8 & 1,18 & 1,608 \\
\hline Ingresos corregidos hombre & 0 & 15911,53 & 672,9941 & 994,4706 \\
\hline Ingresos corregidos mujer & 0 & 18030,36 & 611,5490 & 1061,4378 \\
\hline
\end{tabular}

Tabla 8. Media y desviación típica de las variables cuantitativas en el modelo de salarización. Elaboración propia a partir de IGE (2005), "Enquisa de condicións de vida das familias", año 2003, Metodología 2005

La fórmula anterior es básica para el estudio económico de este modelo. De las 6.363 personas que forman la muestra son 3.767 hombres y 2.596 mujeres.

Pero si queremos analizar la influencia de cada una de las variables cuantitativas, las variables categóricas tenemos que hacerlas fijas (suponemos que partimos de españoles que pertenecen a municipios grandes, casados, que tienen entre 41 y 45 años, y cuyo nivel de estudios es bachillerato y similar). Cogemos estas categorías y no otras porque nos parece que éstas aglutinan a una gran cantidad de personas y que, por tanto, son bastantes representativas.

Si estamos analizando la influencia del número de miembros sobre la probabilidad de trabajar por cuenta ajena procedemos del siguiente modo:

Parámetro Ingresos corregidos hombre/mujer*renta media ingresos corregidos hombre/mujer. Parámetro Número miembros hombre/mujer*valor del número de miembros (variable).

Y, si por el contrario, estamos analizando la influencia de los ingresos corregidos sobre la probabilidad de trabajar por cuenta ajena, resolvemos así:

Parámetro Ingresos corregidos hombre/mujer*valor de ingresos corregidos hombre/mujer (variable). 
Parámetro Número miembros hombre/mujer*número medio miembros hombre/mujer.

Para el estudio económico de las distintas representaciones gráficas de la probabilidad de trabajar por cuenta ajena nos limitaremos a coger únicamente el sector servicios, por varias razones:

- Es el sector económico más numeroso de la población, tanto en hombres como en mujeres.

- Es un sector en el que hay una importante representación tanto en trabajadores por cuenta ajena como por cuenta propia en ambos sexos.

Si nos centramos en los datos correspondientes al sector servicios, tenemos los porcentajes recogidos en la tabla 9.

\begin{tabular}{|c|c|c|}
\hline Sector servicios & Cuenta ajena & Cuenta propia \\
\hline Hombres & $72,66 \%$ & $27,34 \%$ \\
Mujeres & $77,35 \%$ & $22,65 \%$ \\
\hline
\end{tabular}

Tabla 9. Porcentaje de trabajadores por cuenta ajena y por cuenta propia en el sector servicios. Elaboración propia a partir de IGE (2005), "Enquisa de condicións de vida das familias", año 2003, Metodología 2005

Teniendo presente las cifras anteriores, vemos que en el sector servicios el porcentaje de mujeres que trabajan por cuenta ajena aventaja al de hombres, una conclusión que nada tiene que ver con el colectivo que aglutina a todos los sectores.

Resultados. Principales efectos de las características personales sobre el trabajo por cuenta ajena

A continuación, exponemos los principales resultados obtenidos del cálculo de las distintas probabilidades de trabajar por cuenta ajena, acompañándolos de algunas representaciones gráficas que avalan estas conclusiones (todas las representaciones se refieren a la dicotomía hombre-mujer).

- En todas las representaciones gráficas correspondientes a los hombres hay dos puntos de máxima probabilidad que corresponden a las personas sin estudios (analfabetas) y con especialización profesional; sin embargo, las mujeres que presentan mayor probabilidad de trabajar por cuenta ajena tienen estudios de primer ciclo de universidad y especialización profesional.

- Con respecto a la relación entre el nivel de estudios y la probabilidad de trabajar por cuenta ajena en los hombres, podemos hablar de la existencia de un primer tramo (hombres sin estudios hasta los que tienen estudios primarios completos) en el que la relación es inversa; a partir de esta categoría, la relación sigue una tendencia creciente. 
- Por tanto, los hombres que tienen estudios primarios completos son los que tienen menor probabilidad de trabajar por cuenta ajena, o dicho de otro modo, los que tienen mayor probabilidad de trabajar por cuenta propia. Estos primeros resultados se observan en el gráfico 1.

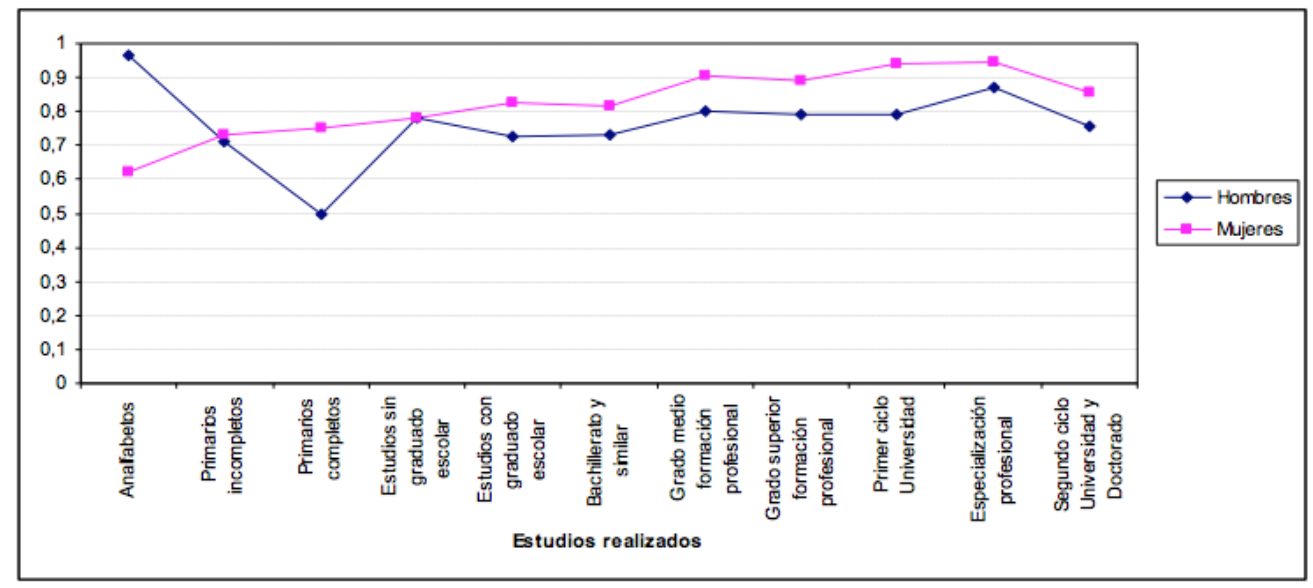

Gráfico 1. Influencia del nivel de estudios sobre la probabilidad de trabajar por cuenta ajena: españoles, municipios grandes, casados, 36-40 años

- En el sexo femenino, la relación entre el nivel de estudios y la probabilidad de trabajar por cuenta ajena es básicamente creciente. Esta relación se rompe en segundo ciclo de universidad y doctorado, esta excepción afecta además a los dos sexos.

- A mayor edad, la probabilidad de que un hombre y una mujer trabajen por cuenta ajena va disminuyendo. La relación es claramente decreciente, y esta caída es mayor si el nivel de estudios es más bajo (gráfico 2).

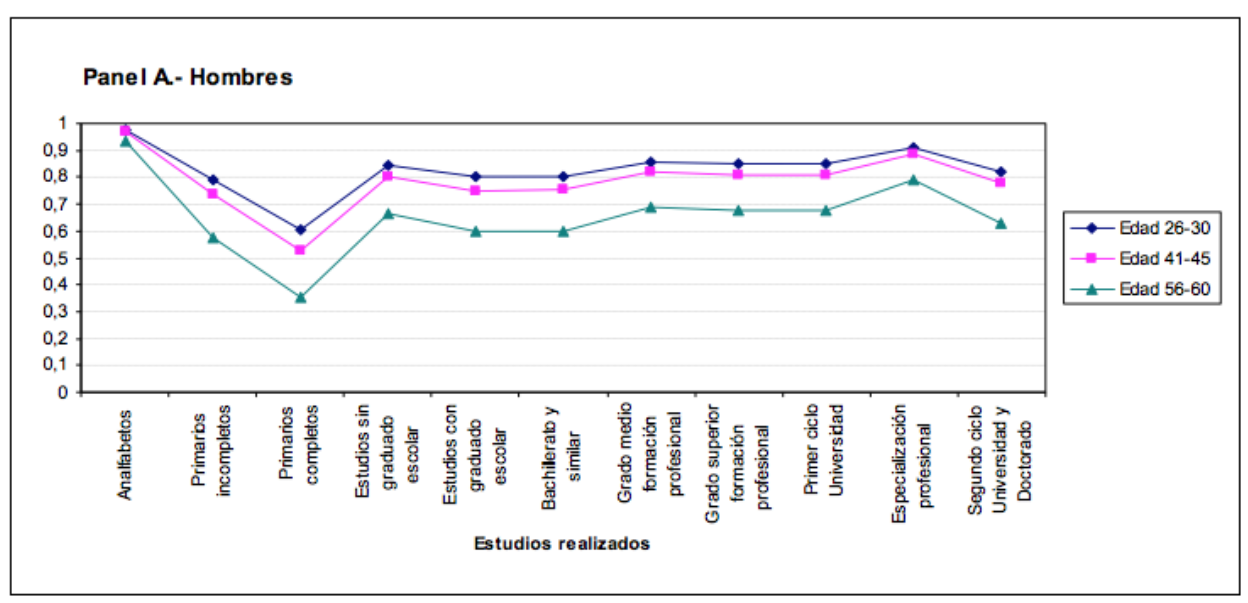




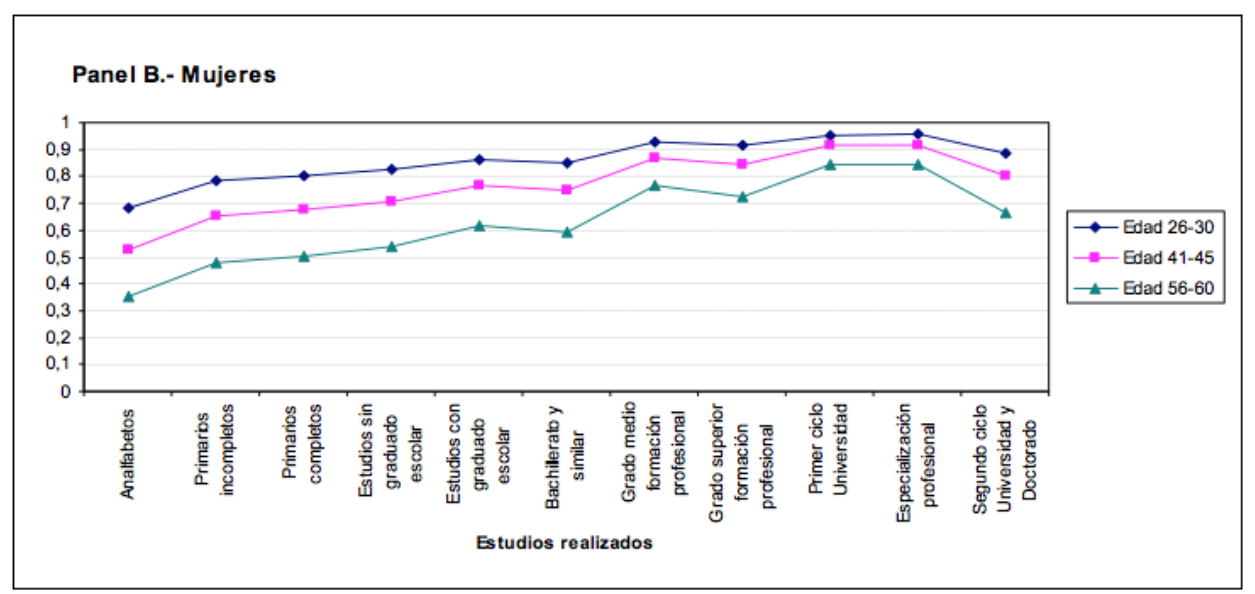

Gráfico 2. Influencia del nivel de estudios sobre la probabilidad de trabajar por cuenta ajena para los intervalos $26-30,41-45$ y 56-60 años: españoles, municipios grandes, casados

- El descenso de la probabilidad de trabajar por cuenta ajena es algo más notorio en el grupo de personas de más edad, (51-65 años).

- Resulta interesante el comportamiento tan dispar en hombres y mujeres en la franja de 36-40 años; mientras en los hombres se constata una caída en la probabilidad de trabajar por cuenta ajena, en las mujeres sucede justo lo contrario, es decir, aumenta (véanse estos dos últimos resultados en el gráfico 3).

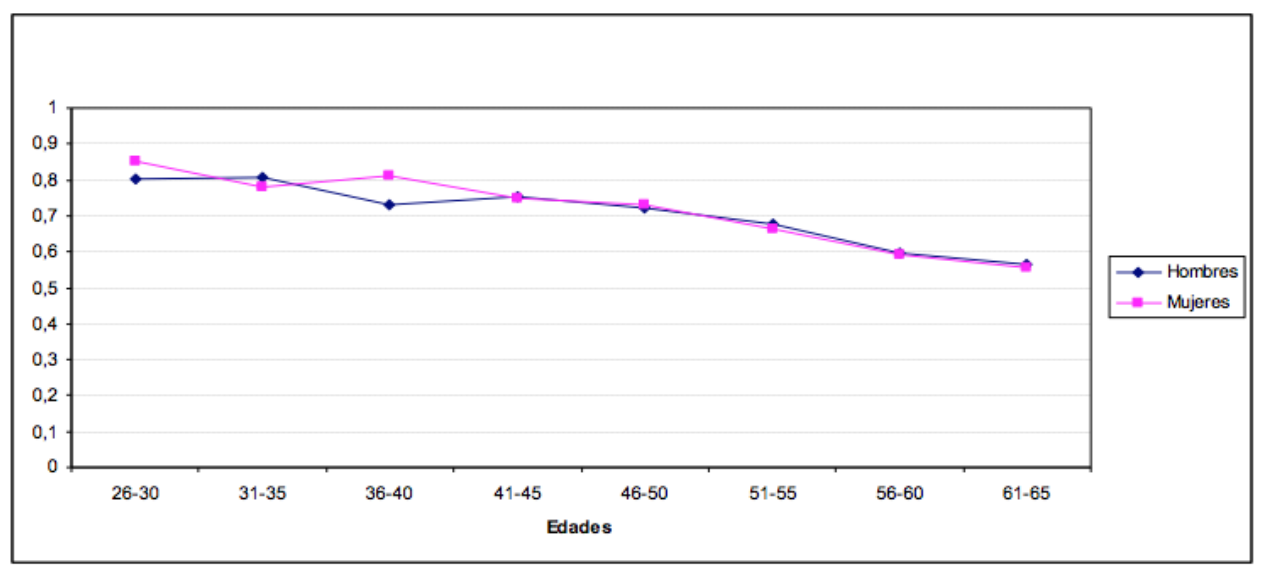

Gráfico 3. Influencia de la edad sobre la probabilidad de trabajar por cuenta ajena: españoles, municipios grandes, casados, estudios bachillerato y similar

- El estado civil de los hombres al que asociamos una menor probabilidad de trabajar por cuenta ajena es el de viudo. Las representaciones de los casados se sitúan en posiciones inferiores en el eje si las comparamos con los solteros (gráfico 4). 


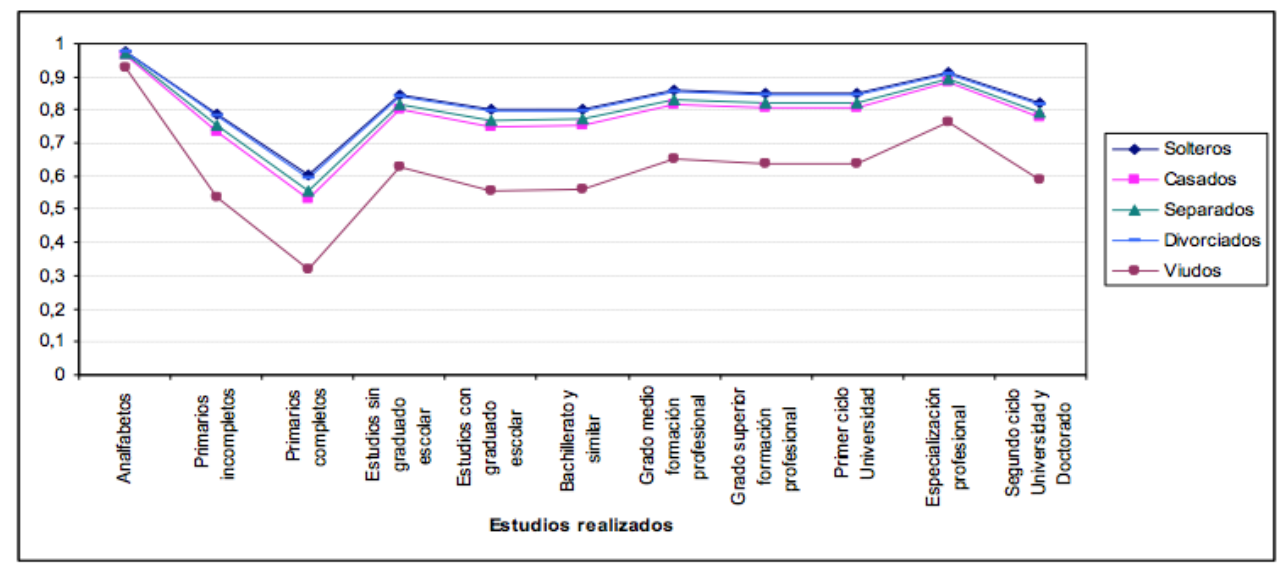

Gráfico 4. Influencia del nivel de estudios sobre la probabilidad de trabajar por cuenta ajena para solteros, casados, separados, divorciados y viudos: españoles, municipios grandes, 41-45 años

- La probabilidad de trabajar por cuenta ajena en los hombres se mantiene invariable para cualquier tamaño familiar mientras que en las mujeres al aumentar los miembros de la familia la probabilidad disminuye (gráfico 5 ).

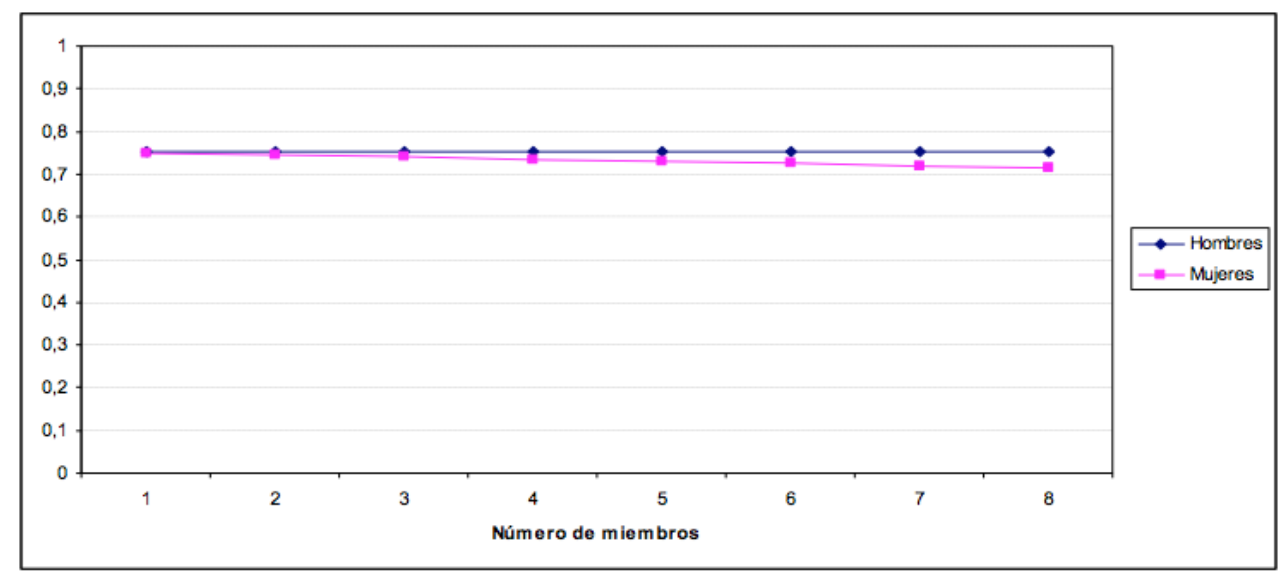

Gráfico 5. Influencia del número de miembros sobre la probabilidad de trabajar por cuenta ajena: españoles, municipios grandes, casados, 41-45 años, bachillerato y similar A continuación, reflejamos en la tabla 10 las variaciones producidas en la probabilidad de trabajar por cuenta ajena para un incremento unitario en el número de miembros, tanto si estudiamos a hombres como si estudiamos a mujeres. 


\begin{tabular}{|c|c|c|}
\hline $\begin{array}{c}\text { Número de } \\
\text { miembros }\end{array}$ & $\begin{array}{c}\text { Variación de la probabilidad de } \\
\text { trabajar por cuenta ajena (\%) } \\
\text { Hombres }\end{array}$ & $\begin{array}{c}\text { Variación de la probabilidad de } \\
\text { trabajar por cuenta ajena (\%) } \\
\text { Mujeres }\end{array}$ \\
\hline $\mathbf{1}$ & & $-0,50$ \\
\hline $\mathbf{2}$ & 0,01 & $-0,50$ \\
\hline $\mathbf{3}$ & 0,01 & $-0,51$ \\
\hline $\mathbf{4}$ & 0,01 & $-0,52$ \\
\hline $\mathbf{5}$ & 0,01 & $-0,52$ \\
\hline $\mathbf{6}$ & 0,01 & $-0,53$ \\
\hline $\mathbf{8}$ & 0,01 & $-0,54$ \\
\hline
\end{tabular}

Tabla 10. Variaciones en la probabilidad de trabajar por cuenta ajena para un incremento unitario en el número de miembros

Si la unidad familiar pasa de tener uno a dos miembros, la probabilidad de que un hombre trabaje por cuenta ajena se incrementa un 0,01\%, mientras que bajo esta misma circunstancia, la probabilidad de que una mujer trabaje por cuenta ajena disminuye alrededor de $0,5 \%$. Vemos que estas variaciones son muy pequeñas, pero en cualquier caso, en los hombres son siempre positivas y constantes, mientras que en las mujeres tienen signo negativo.

- Con independencia del sexo, ante un incremento en los ingresos corregidos familiares, la probabilidad de trabajar por cuenta ajena disminuye (se mueve en la franja del 70-80\%). Estas disminuciones son de escasa cuantía.

Aunque la variable de ingresos corregidos tiene un valor mínimo de 0 y un máximo de 18.030, a la hora de su tratamiento representamos solamente el intervalo $[0,4800]$ ya que de esta manera englobamos el $99 \%$ de los ingresos (gráfico 6 ).

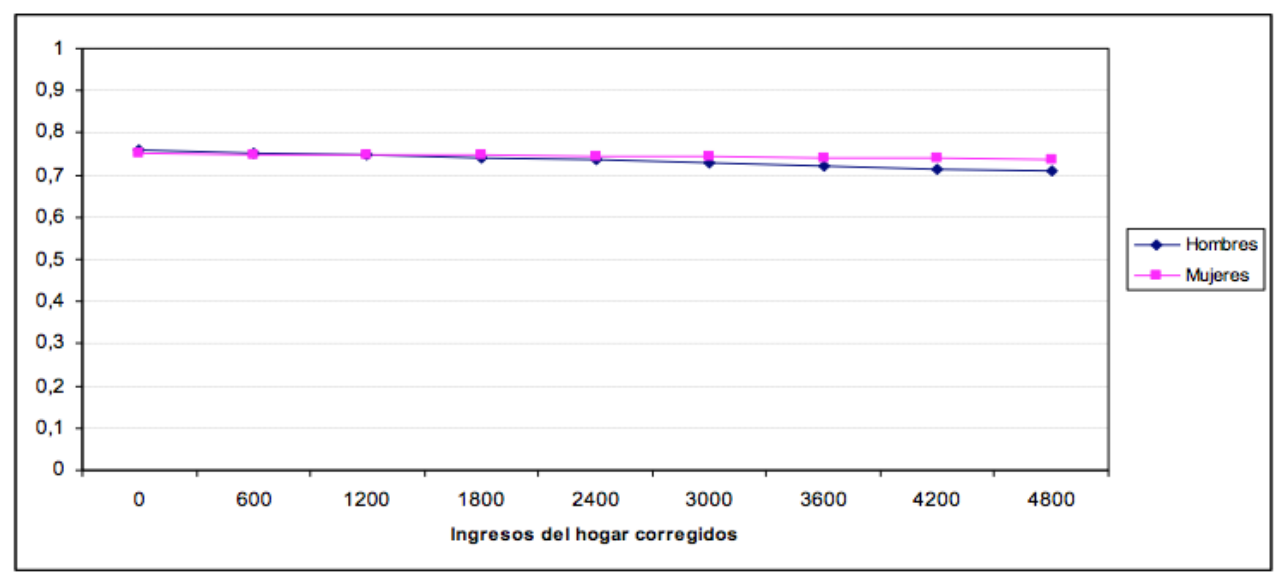

Gráfico 6. Influencia de los ingresos corregidos sobre la probabilidad de trabajar por cuenta ajena: españoles, municipios grandes, casados, 41-45 años, bachillerato y similar Continuamos con el estudio detallado de la influencia de los ingresos del hogar corregidos en las distintas probabilidades. En la tabla 11 aparecen las variaciones en la probabilidad de trabajar por cuenta ajena para un incremento de 600 euros en los ingresos. 


\begin{tabular}{|c|c|c|}
\hline $\begin{array}{c}\text { Ingresos } \\
\text { del hogar } \\
\text { corregidos }\end{array}$ & $\begin{array}{c}\text { Variación de la probabilidad de } \\
\text { trabajar por cuenta ajena (\%) } \\
\text { Hombres }\end{array}$ & $\begin{array}{c}\text { Variación de la probabilidad de } \\
\text { trabajar por cuenta ajena (\%) } \\
\text { Mujeres }\end{array}$ \\
\hline 0 & & $-0,16$ \\
\hline 600 & $-0,60$ & $-0,16$ \\
\hline 1200 & $-0,61$ & $-0,16$ \\
\hline 1800 & $-0,62$ & $-0,16$ \\
\hline 2400 & $-0,63$ & $-0,16$ \\
\hline 3000 & $-0,64$ & $-0,16$ \\
\hline 3600 & $-0,65$ & $-0,16$ \\
\hline 4200 & $-0,66$ & $-0,16$ \\
\hline 4800 & $-0,67$ & . \\
\hline
\end{tabular}

Tabla 11. Variaciones en la probabilidad de trabajar por cuenta ajena para un incremento de 600 euros en los ingresos del hogar corregidos

Igualmente en esta ocasión, reflejamos variaciones de escasa cuantía. Observamos en las mujeres disminuciones de probabilidad constantes $(0,16 \%)$, y casi constantes en el caso de los hombres (en torno a $0,60 \%$ ).

\section{Conclusiones}

En palabras del Servicio Galego de Igualdade de la Xunta de Galicia en su publicación "As mulleres galegas no século XX" (1999): Es fundamental concienciar a la sociedad, tanto a hombres como a mujeres de la necesidad de conciliar la vida familiar con la profesional, implicando a unas y otros en la participación compartida e igualitaria de las responsabilidades familiares y laborales. El sistema educativo es uno de los instrumentos básicos para corregir las desigualdades sociales, entre ellas las que se generan por razón del sexo. Es conveniente seguir desarrollando políticas que permitan los cambios sociales necesarios para que la igualdad, reconocida legalmente, se transforme en una igualdad efectiva y real.

La mujer está desempeñando un papel fundamental en la sociedad actual. La mujer ha trabajado casi siempre fuera de casa: en el campo, en las fábricas o en los servicios. Lo diferente es que en la actualidad lo hace de forma masiva, en mejores condiciones, en términos de formación más preparada y en mejores puestos.

Tres hechos observables son:

- Las desigualdades en género se van reduciendo con el paso del tiempo,

- La población con estudios universitarios encuentra más facilidades en el mercado de trabajo que la población sin esos estudios,

- A mayor nivel de estudios, las diferencias laborales entre hombres y mujeres se van haciendo menores (planteamiento teórico que comprobamos empíricamente).

Una vez revisadas las distintas teorías que relacionan mercado de trabajo y género, el objetivo de nuestro análisis se ha centrado en el cálculo de las distintas probabilidades de trabajar por cuenta ajena en la dualidad hombre-mujer, estableciendo para ello distintas premisas. 
En nuestra investigación vimos que nuestro modelo de salarización es el mejor si lo comparamos con otros hipotéticos modelos (llamados modelos restringidos) en los que las variables explicativas aparecen sin interactuar con el sexo. Este hecho justifica la forma de construir todas las variables explicativas, y en último término, la importancia que le hemos dado inicialmente a la influencia del género en el mercado de trabajo.

La definición, cálculo y justificación de la variable ingresos del hogar corregidos es una de las aportaciones novedosas de este trabajo.

En las representaciones gráficas de las probabilidades de trabajar por cuenta ajena pudimos apreciar diferente comportamiento en función del género de la persona analizada. Concluimos que a mayor edad, la probabilidad de que un hombre y una mujer trabajen por cuenta ajena va disminuyendo. La probabilidad de trabajar por cuenta ajena en los hombres se mantiene invariable para cualquier tamaño familiar, mientras que en las mujeres al aumentar los miembros de la familia la probabilidad disminuye. Ante un incremento en los ingresos corregidos familiares, la probabilidad de trabajar por cuenta ajena disminuye en hombres y mujeres, aunque los porcentajes son distintos en función del género (se mueven en la franja del 70-80\%); estas disminuciones son de escasa cuantía.

Los resultados obtenidos del análisis empírico los consideramos válidos en un momento económico de crecimiento sostenido. En un período recesivo como el actual, somos escépticos de su validez. Una nueva vía de estudio consiste en averiguar si el ciclo económico hace variar las conclusiones de esta investigación.

Por todo ello, el mercado de trabajo tanto teóricamente como desde el punto de vista de investigación aplicada plantea un interesante campo de debate del que se pueden extraer conclusiones de gran relevancia y de indudable actualidad.

\section{Referencias}

AGRESTI, A. (1996). An introduction to categorical data analysis. New York: Wiley.

ALBERT, C.; JUÁREZ, J.P.; SÁNCHEZ, R.; TOHARIA, L. (2003). Del sistema educativo al mercado de trabajo: un análisis de flujos, Revista de Educación, 330: 137-155.

AMEMIYA, T. (1981). Qualitative response model: a survey, Journal of Economic Literature, 19: 481-536.

ASOCIACIÓN MUJERES Y TECNOLOGÍA (2007). Jornadas de emprendizaje. El autoempleo como alternativa de inserción laboral. http://www.eniac.org.es. (Fecha último acceso: 6 de Junio de 2008). 
BARREIRO GARCÍA, J.S.; MARTÍNEZ SEIJAS, M.P. (2006). Mercado de trabajo e inserción social femenina. http://www.monografias.com/trabajos34/ mercado-trabajo/mercado-trabajo.html. (Fecha último acceso: 16 de septiembre de 2011)

BECKER, G. (1962). Investment in human capital: a theoretical analysis, The Journal of Political Economy, 70(5): 9-49. http://dx.doi.org/10.1086/258724

BECKER, G. (1971). The economics of discrimination. Chicago: University Press. http://dx.doi.org/10.7208/chicago/9780226041049.001.0001

BECKER, G. (1985). Human Capital, effort and the sexual division of labour, Journal of Labor Economics, 3: 33-58. http://dx.doi.org/10.1086/298075

BULOW, J.; SUMMERS, L. (1986). A theory of dual labor markets with application to industrial policy, discrimination and keynesian unemployment, Journal of Labor Economics, 4: 376-414. http://dx.doi.org/10.1086/298116

BUTTNER, H.; MOORE, D. (1997). Women organizational exodus to entrepreneurship: selfreported motivations and correlates with success, Journal of Small Business Management, 34-46.

CÁCERES RUIZ, J.I.; ESCOT, L.; FERNÁNDEZ, J.A.; SAIZ, J. (2004). La segregación ocupacional y sectorial de la mujer en el mercado de trabajo español. Documentos de Trabajo de la Facultad de Ciencias Económicas y Empresariales de la Universidad Complutense de Madrid.

CALVET-PUiG, M.D.; LUSA-GARCIA, A.; MARTÍnEZ-COSTA, C.; PONS, O.; TURA-SOLVAS, M. (2009). Planes de igualdad por ley: Ventajas de igualdad de oportunidades entre mujeres y hombres para las empresas Intangible Capital, 5(2): 169-182.

COX, D.R.; SNELL, E.J. (1989). The analysis of Binary Data. Londres: Chapman y Hall.

DEL RÍO OTERO, C. (2003). ¿Existe discriminación de xénero no mercado de traballo?, en Xosé H. Vázquez-Vicente (ed.) Vigo. Economía e Sociedade (pp. 307-332). Edicións Xerais de Galicia. Vigo.

FREIRE SEOANE, M.J.; TEIJEIRO ALVAREZ, M. (2009). Análisis de los factores que afectan a la decisión de ser emprendedor, Cuadernos de Economía, 32(90): 5-28.

FRUTOS BALIBREA, L.; TITOS GIL, S. (2001). Formación y trabajo autónomo desde la perspectiva de género. X Jornadas de la Asociación de Economía de la Educación, Murcia: 309-320.

GATEWOOD, E.J.; SHAVER, K.G.; GARTNER, W.B. (1995). A longitudinal study of cognitive factors influencing start-up behaviours and success at venture creation, Journal of Business Venturing, 10(5): 371-391. http://dx.doi.org/10.1016/0883-9026(95)00035-7 
GOLDIN, C. (1986). Monitoring costs and occupational segregation by sex: An historical analysis, Journal of Labor Economics, 4: 1-27. http://dx.doi.org/10.1086/298091

GRADÍN, C.; OTERO, M.; ARÉVALO, R. (2003). Elementos explicativos de la desigualdad en Galicia. Género, Mercado de Trabajo y Vivienda. Monografía 18. Instituto de Estudios Económicos de Galicia- Pedro Barrié de la Maza.

GUZMÁN CUEVAS, J.; RODRÍGUEZ GUTIERREZ, M.J. (2008). Comportamiento de las mujeres empresarias: una visión global, Revista de Economía Mundial, Universidad de Huelva, 18: 381-392.

HARTMANN, H. (1994). Capitalismo, patriarcado y segregación de los empleos por sexos. En Borderias, C. et al., Las mujeres y el trabajo: rupturas conceptuales (pp. 253-294). Icaria y Fuhem. Barcelona.

HOSMER, D.W.; LEMESHOW, S. (1989). Applied Logistic Regression. New York: Wiley.

IGE (2005). Enquisa de condicións de vida das familias. Ano 2003. Metodoloxía. Mimeo: Santiago de Compostela.

IVIE (2008a). Capital humano y ciclo vital en España, Cuadernos de Capital Humano, 90.

IVIE (2008b). Diferencias salariales ligadas al nivel educativo, Cuadernos de Capital Humano, 92.

LAMAS, M. (2000). El Género. La construcción cultural de la diferencia sexual. Programa Universitario de Estudios de Género. UNAM. México.

LARRAÑAgA SARRIEGUI, M. (2000). Análisis teóricos de la desigualdad. Area temática 3. Economía feminista. http://www.ucm.es/info/ec/jec7/pdf/com3-6.pdf. (Fecha último acceso: 7 de marzo de 2012).

MARTIN, J.; ROBERTS, C. (1984). Women and work: a lifetime perspective. London: HSMO.

MURGATROYD, L. (1982). Gender and Occupational Stratification, The Sociological Review, 30(4): 574-602. http://dx.doi.org/10.1111/j.1467-954X.1982.tb00669.x

OGUIZA, A.; GALLASTEGUI, I.; NÚÑEZ, V. (2004). La población ocupada en la CAPV (19931999). Género y formación como características relevantes, Estadística Española, 46(156): 229-292.

RIOBÓO LESTÓN, I.; MARTÍN LÓPEZ, C. (2011). Género y mercado de trabajo en Galicia. Análisis de equidad mediante indicadores sintéticos, Revista Galega de Economía, 20: 1-13.

ROSENER, J. (1990). Ways women lead, Harvard Business Review: 119-125.

SÁEZ LARA, C. (1994). Mujeres y mercado de trabajo. Las discriminaciones directas e indirectas. Consejo Económico y Social, Madrid. 
SÁNCHEZ MORENO, E.; DELICADO LOSA, C. (2007). Mujeres, juventud y mercado de trabajo en España. Consejo de la Juventud de España, Madrid.

SÁNCHEZ SELLERO, M.C. (2010): Factores determinantes para la inserción y distintas formas de participación en el mercado laboral gallego. Tesis Doctoral, Universidade da Coruña.

SERVICIO GALEGO DE IGUALDADE (1999). As mulleres galegas no século $X X$. Consellería de familia e promoción do emprego, muller e xuventude. Santiago de Compostela: Xunta de Galicia.

Intangible Capital, 2013 (www.intangiblecapital.org)

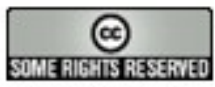

El artículo está con Reconocimiento-NoComercial 3.0 de Creative Commons. Puede copiarlo, distribuirlo y comunicarlo públicamente siempre que cite a su autor y a Intangible Capital. No lo utilice para fines comerciales. La licencia completa se puede consultar en http://creativecommons.org/licenses/by-nc/3.0/es/ 